\title{
CHARACTERIZATION OF THE FORCES, POWERS AND EFFICIENCY FOR A NAVAL HYDROELECTROMAGNETIC THRUSTER
}

\author{
Vasile DOBREF, Petrică POPOV \\ "Mircea cel Bătrân”Naval Academy, Constanța, Romania \\ vasile.dobref@anmb.ro, petrica.popov@anmb.ro
}

\begin{abstract}
This paper presents a theoretical model of the hydroelectromagnetic thruster (electromagnetic three-phase induction seawater pump), which was developed from a semi-space model for an infinity conductor, where the electrically fluid - seawater occupies a semi-space, bordered by an ideally ferromagnetic wall. At the fluid surface, it is found a web of currents having density vector oriented in the direction of $O y$ axis, and fluid movement - considered a translational motion at constant velocity - is directed along the Ox axis. In this regard, for certain arrangements of three phase windings inductors, were performed calculations in order to estimate the forces, powers and efficiency for electromagnetic three-phase induction pump, having the seawater as induced fluid. Following the analysis of the traction force expression, it was found that it is possible to achieve a hydroelectromagnetic thruster, as a rectangular channel with the specific configuration of the threephase windings, enabling to develop the electromagnetic traction forces for seawater as fluid induced conductor, forces comparable with propellers systems
\end{abstract}

\section{Keywords: hydro-electromagnetic, induction pump}

\section{Introduction}

The possibility of getting the electromagnetic pump effect for an electrolyte - seawater, was analyzed for a parallelepiped canal with rectangular section. It is considered a long enough channel because we have to neglect the end effects, with a rectangular section of $\mathrm{S}=2 \mathrm{~b}$ $\times 2 \mathrm{c}$, having the walls located in $\mathrm{z}= \pm \mathrm{c}$ plans made of insulated thin material, and the walls located in $y= \pm b$ plane made of perfect conductive material $(\sigma=\infty)$. Outside the channel the medium is ideal ferromagnetic (with permeability of $\mu_{\mathrm{Fe}}=$ $\infty$ ), and a fluid conductor (seawater) with known $\varepsilon, \mu, \sigma$, is running through the channel with the speed $\mathrm{v}$ [1]. There is a web of currents at the surface of the conductor fluid. The three-phase wrappings of the inductor can be disposed in two ways, as shown in fig. I. These two types of wrappings are called A and B (type A wrapping - distributed wrapping; type B wrapping - ring wrapping) having different types of boundary condition (Fig. 1):

$$
\underline{H}_{x}(x, c)=-\underline{J}_{S} ; \quad \underline{H}_{x}(x,-c)=\underline{J}_{S}
$$

- for the channel with type A wrapping

$$
\underline{H}_{x}(x, c)=-\underline{J}_{S} ; \quad \underline{H}_{x}(x,-c)=-\underline{J}_{S}
$$

- for the channel with type B wrapping

In the conductive walls, the tangential component of the intensity of the electric field is null. Under these circumstances, the electromagnetic field in the two channels $A$ and $B$ can be determined [2]. 
2. The electromagnetic field calculation in type $A$ and $B$ wrapping

- Type A wrapping - distributed wrapping. For the calculation of the electromagnetic field the constants $\mathrm{C}_{1}$ and $\mathrm{C}_{2}$ are determined considering the limit conditions, solving the system:
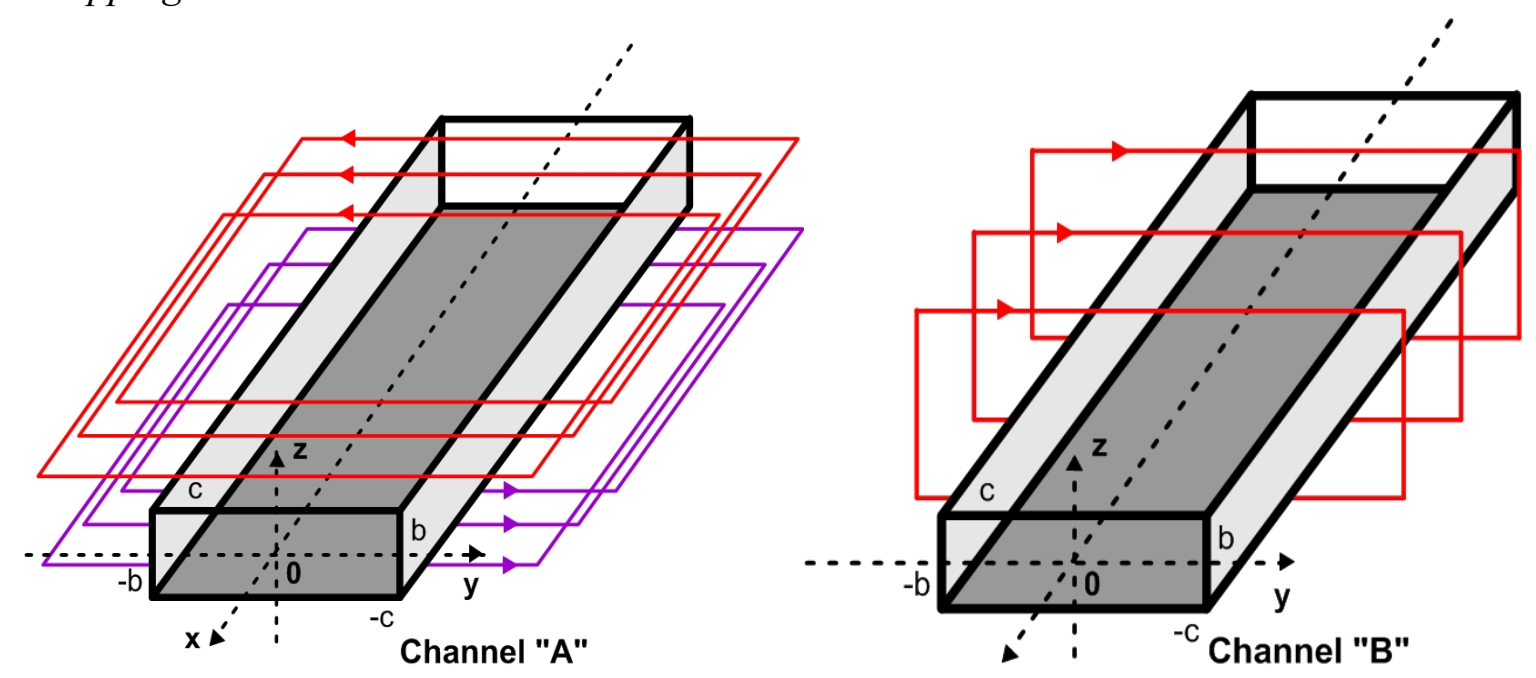

Figure 1. Two possibilities of inductive three-phase wrapping disposals: channel " $A$ "-distributed wrapping, channel " $B$ "-ring type wrapping

$-\mu J_{s_{o}}=C_{1} e^{\frac{\gamma}{\delta} c}+C_{2} e^{-\frac{\gamma}{\delta} c}$

$\mu J_{s_{o}}=C_{1} e^{-\frac{\gamma}{\delta} c}+C_{2} e^{\frac{\gamma_{c}}{\delta}}$

Results:

$$
C_{1}=-C_{2}=-\frac{\mu J_{S_{o}}}{2 \operatorname{sh} \frac{\gamma}{\delta} c}
$$

The component on the OX axis of the magnetic inductor is:

$$
\underline{B}_{x}=-\left[\mu J_{S_{o}}\right] \frac{\operatorname{sh} \gamma z^{\prime}}{s h \gamma c^{\prime}} \cdot e^{-j \alpha x^{\prime}}
$$

Using the relation (6) the current density is determined:

$$
\begin{gathered}
\underline{J}_{Y}(x, z)=\underline{J}(x, z) \\
\underline{B}_{z}=-j\left[\mu J_{s_{o}}\right] \cdot \frac{\alpha}{\gamma} \cdot \frac{c h \gamma z^{\prime}}{s h \gamma c^{\prime}} \cdot e^{-j \alpha x^{\prime}} \\
\underline{J}=-\left[\frac{J_{s_{o}}}{\delta}\right] \cdot 2 j s \cdot \frac{1}{\gamma} \cdot \frac{c h \gamma z^{\prime}}{s h \gamma c^{\prime}} \cdot e^{-j \alpha x^{\prime}}
\end{gathered}
$$

- $\quad$ Type $B$ wrapping - ring wrapping. The constants $C_{1}$ and $C_{2}$ are determined considering the limit conditions:

$$
C_{1}=-C_{2}=-\frac{\mu J_{S o}}{2 \operatorname{ch} \frac{\gamma}{\delta} c}
$$

The components on the OX axis of the magnetic induction and the current density are:

$$
\begin{aligned}
& \underline{B}_{x}=-\left[\mu J_{S o}\right] \frac{c h \gamma z^{\prime}}{c h \gamma c^{\prime}} e^{-j \alpha x^{\prime}} \\
& \underline{B}_{x}=-j\left[\mu J_{S_{0}}\right] \frac{\alpha}{\gamma} \frac{s h \gamma z^{\prime}}{c h \gamma c^{\prime}} e^{-j \alpha x^{\prime}} \\
& \underline{J}=-\frac{\mu J_{S_{0}}}{\delta} \cdot \frac{2 j s}{\gamma} \cdot \frac{s h \gamma z^{\prime}}{c h \gamma c^{\prime}} \cdot e^{-\mathrm{j} \alpha \mathrm{x}^{\prime}}
\end{aligned}
$$

3. The calculation of forces, power and efficiency in the type $A$ and $B$ wrapping channel.

- Type A wrapping - distributed wrapping. For the medium density forces $\overline{\widetilde{\mathrm{f}}_{\mathrm{x}}}$ and $\overline{\widetilde{\mathrm{f}}_{z}}$ are obtained the following expression: 


$$
\begin{aligned}
& \overline{\widetilde{f}_{x}}=\operatorname{Re}\left(\underline{J} \cdot \underline{B}_{z}^{*}\right) \cdot \bar{u}_{x} \\
& \widetilde{\widetilde{f}}_{x}=\frac{\mu J_{S_{o}}^{2}}{\delta} \cdot \frac{2 \alpha s}{\varepsilon} \cdot \frac{\operatorname{ch} \theta_{1} z^{\prime}+\cos \theta_{2} z^{\prime}}{\operatorname{ch} \theta_{1} c^{\prime}-\cos \theta_{2} c^{\prime}} \cdot \bar{u}_{x} \\
& \overline{\widetilde{f}_{z}}=-\operatorname{Re}\left(\underline{J} \cdot \underline{B}_{X}^{*}\right) \cdot \bar{u}_{z} \\
& \widetilde{\widetilde{f}}_{z}=-\frac{\mu J_{S_{o}}^{2}}{\delta} \cdot \frac{s}{\varepsilon} \cdot \frac{\operatorname{sh} \theta_{1} z^{\prime}+\theta_{1} \sin \theta_{2} z^{\prime}}{\operatorname{ch} \theta_{1} c^{\prime}-\cos \theta_{2} c^{\prime}} \cdot \bar{u}_{z}
\end{aligned}
$$

The resulted medium power that actions on the ax Ox on a fluid volume $2 b \times 2 c \times \lambda$ the following equation is given [3]:

$$
\overline{\widetilde{F}}_{X}=\mu J_{S_{0}}^{2} \cdot 4 b c \cdot 4 \pi \cdot \frac{s}{\varepsilon c^{\prime}} \cdot \frac{\frac{\operatorname{sh} \theta_{1} c^{\prime}}{\theta_{1}}+\frac{\sin \theta_{2} c^{\prime}}{\theta_{2}}}{\operatorname{ch} \theta_{l} c^{\prime}-\cos \theta_{2} c^{\prime}} \cdot \bar{u}_{x}
$$

Because $\theta_{1} \cdot \theta_{2}=4 \mathrm{~s}$, and writing with the adimensional function:

$$
\mathfrak{I}=\frac{\pi}{\varepsilon c^{\prime}} \cdot \frac{\theta_{2} \operatorname{sh} \theta_{1} c^{\prime}+\theta_{1} \sin \theta_{2} c^{\prime}}{\operatorname{ch} \theta_{1} c^{\prime}-\cos \theta_{2} c^{\prime}}
$$

of variables $\alpha$, s and c', the equation (16) becomes:

$$
\overline{\widetilde{F}}_{X}=\mu J_{s_{0}}^{2} \cdot 4 b c \cdot \Im\left(\alpha, s, c^{\prime}\right) \cdot \bar{u}_{x}
$$

It is shown that the force $\overline{\widetilde{F}}_{X}$ is proportional to the reference pressure $\mu J_{s_{0}}^{2}$, with the transversal section $4 b c$ and with the expression $\mathfrak{I}$. For a given channel and conductor, the power varies depending on slipping, as the function of $\mathfrak{I}$.

The medium resultant force, perpendicular the moment direction, is null. The subpressure at the channel limits $\mathrm{z}= \pm \mathrm{c}$ as far as the axial plan for $\mathrm{z}=0$, is:

$$
\begin{aligned}
& \Delta p=\widetilde{p}(x, 0)-\tilde{p}(x, c)=\int_{0}^{c} \widetilde{f}_{Z} \cdot d z, \\
& \Delta p=\mu J_{s_{0}}^{2}\left(\frac{1}{2}-\frac{\alpha^{2}}{2 \varepsilon} \cdot \frac{\operatorname{ch} \theta_{I} c^{\prime}+\cos \theta_{2} c^{\prime}-2}{\operatorname{ch} \theta_{I} c^{\prime}-\cos \theta_{2} c^{\prime}}\right)
\end{aligned}
$$

So, the electromagnetic forces tend to take off the fluids on the walls.

The useful power and the lost power are calculated for the fluid volume trough the Joule - Lenz effect: $2 b \times 2 c \times \lambda$ :

$$
\widetilde{P}_{u}=\widetilde{F}_{x} \cdot v=\mu J_{s_{0}}^{2} \cdot 4 b c \cdot v \cdot \mathfrak{I}
$$

$\widetilde{P}_{j}=\operatorname{Re} \iiint \underline{\underline{E}} \cdot \bar{J}^{*} d v=\mu J \cdot 4 b c \cdot s \cdot v_{0} \cdot \mathfrak{I}(22)$

It's shown that the theoretic efficiency of the change of the electromagnetic field in mechanical energy is $\eta=\mathrm{v} / \mathrm{v}_{0}=1$-s.

- Type B wrapping - ring wrapping. For the medium density forces $\overline{\widetilde{\mathrm{f}}_{\mathrm{x}}}$ and $\overline{\widetilde{\mathrm{f}}_{\mathrm{z}}}$ the following expression are obtained:

$\overline{\widetilde{f}_{x}}=\frac{\mu J_{S_{o}}^{2}}{\delta} \cdot \frac{2 \alpha s}{\varepsilon} \cdot \frac{\operatorname{ch} \theta_{1} z^{\prime}-\cos \theta_{2} z^{\prime}}{\operatorname{ch} \theta_{1} c^{\prime} ;+\cos \theta_{2} c^{\prime}} \cdot \bar{u}_{x}$

$\overline{\widetilde{f}_{z}}=\frac{\mu J_{S_{o}}^{2}}{\delta} \cdot \frac{s}{\varepsilon} \cdot \frac{\operatorname{sh} \theta_{1} z^{\prime}-\theta_{1} \sin \theta_{2} z^{\prime}}{\operatorname{ch} \theta_{1} c^{\prime}+\cos \theta_{2} c^{\prime}} \cdot \bar{u}_{z}$

The resulted medium power that actions on the ax Ox on a fluid volume $2 b \times 2 c \times \lambda$ is given by the following equation [3]:

$$
\overline{\widetilde{F}}_{X}=\mu J_{s_{0}}^{2} \cdot 4 b c \cdot \Gamma\left(\alpha, s, c^{\prime}\right) \quad \bar{u}_{x}
$$

Where, $\Gamma\left(\alpha, s, c^{\prime}\right)$ is:

$$
\Gamma=\frac{\pi}{\varepsilon c^{\prime}} \cdot \frac{\theta_{2} \operatorname{sh} \theta_{1} c^{\prime}-\theta_{1} \sin \theta_{2} c^{\prime}}{\operatorname{ch} \theta_{1} c^{\prime}+\cos \theta_{2} c^{\prime}}
$$

The medium resultant perpendicular the moment direction is null. The sub pressure at the channel limits $\mathrm{z}= \pm \mathrm{c}$ as far as the axial plan is concerned $\mathrm{z}=0$ is:

$$
\Delta p=\tilde{p}(x, 0)-\tilde{p}(x, c)=\int_{0}^{c} \widetilde{f}_{Z} \cdot d z
$$

$$
\Delta p=\mu J_{S_{0}}^{2} \cdot\left(\frac{1}{2} \cdot \frac{\alpha^{2}}{2 \varepsilon} \cdot \frac{\operatorname{ch} \theta_{1} c^{\prime}-\cos \theta_{2} c^{\prime}-2}{\operatorname{ch} \theta_{1} c^{\prime}+\cos \theta_{2} c^{\prime}}\right)
$$

So, the electromagnetic forces tend to take off the fluids on the walls.

The useful power and the lost power are calculated for the fluid volume trough the Joule - Lenz effect: $2 b \times 2 c \times \lambda$ :

$$
\begin{aligned}
\widetilde{P}_{u} & =\mu J_{s_{0}}^{2} \cdot 4 b c \cdot v \cdot \Gamma \\
\widetilde{P}_{j} & =\mu J \cdot 4 b c \cdot s \cdot v_{0} \cdot \Gamma \\
\eta & =\mathrm{v} / \mathrm{v}_{0}=1-\mathrm{s}
\end{aligned}
$$

4. Results and discussion. The function $\mathrm{T}_{\mathrm{s}}(\mathrm{s})$ is represented in figure 2 , for four wavelengths of the progressive field and varied values of frequency, considering the 
seawater as conductive fluid

The penetration depth $\delta$ was calculated with the relation:

$$
\delta=\sqrt{\frac{2}{\omega \mu \sigma}}
$$

We have not interested in penetration depths bigger than $(\delta \geq \mathrm{c})$, graphic representation were made for $\delta<8 \mathrm{~m}$

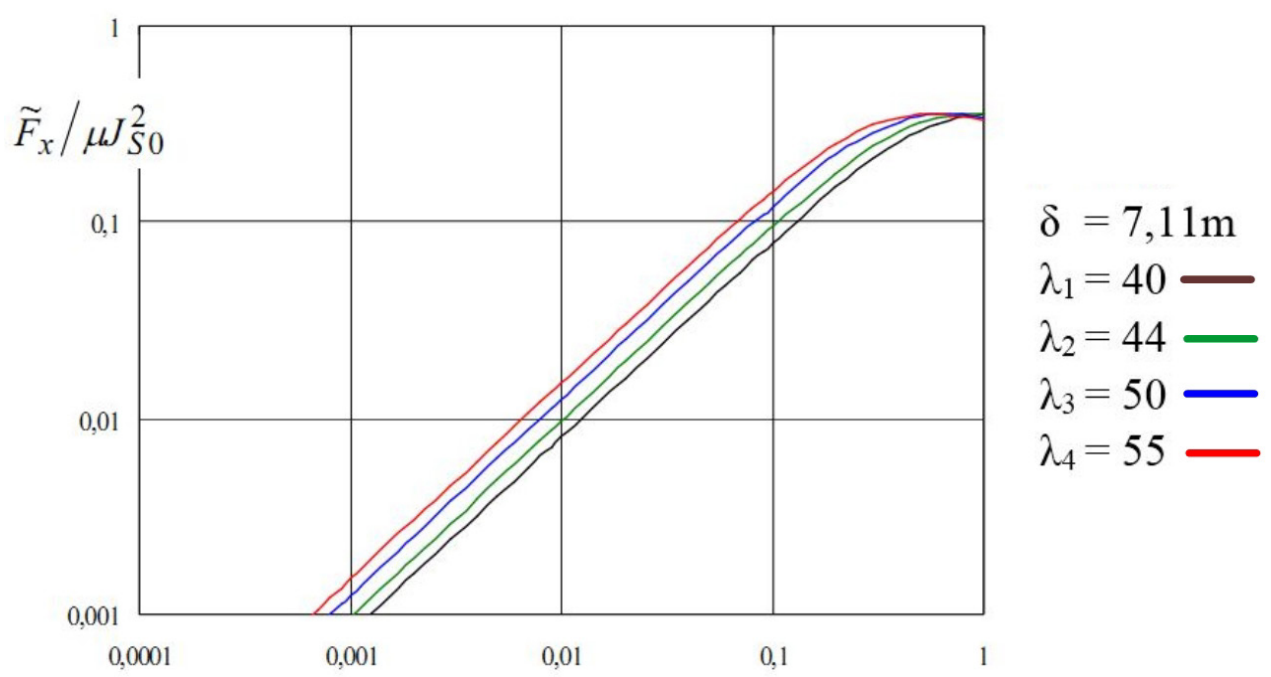

Figure 2. The variation's function $T_{s}=\overline{\widetilde{F}}_{X} / \mu J_{s_{0}}^{2}$ for 4 wave length to progressive field

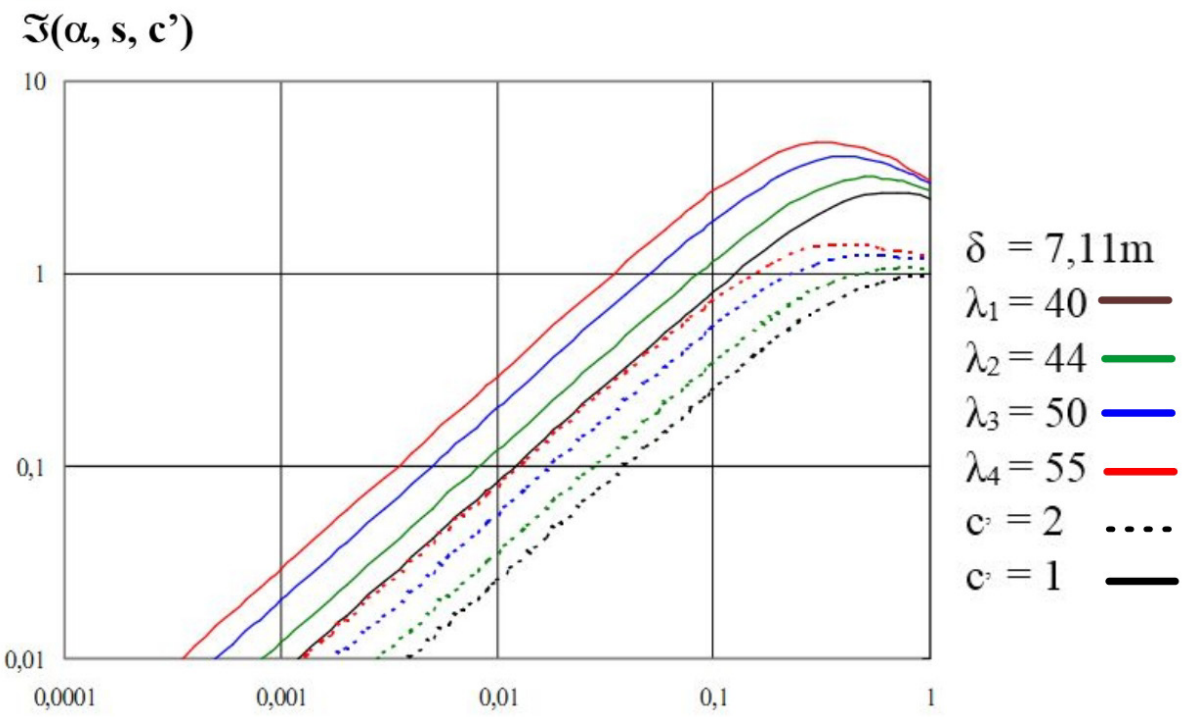

Figure 3. The variation's function $\mathfrak{I}\left(\alpha, \mathrm{s}, \mathrm{c}^{\prime}\right)$ for 4 wave length to progressive field

For large values of $\mathrm{c}^{\prime}\left(\mathrm{c}^{\prime}=2\right)$, the functions $\mathfrak{I}\left(\alpha, \mathrm{s}, \mathrm{c}^{\prime}\right)$ and $\Gamma\left(\alpha, s, c^{\prime}\right)$ tend to overlap.

The type A channel has more advantages than channel $B$. When the wavelength (numeric simulations), taking into account the reasonable sizes of the hydroelectromagnetic thruster.

For the same conductive fluid - seawater, was represented the equation $\mathfrak{I}\left(\alpha, \mathrm{s}, \mathrm{c}^{\prime}\right)$, for the same wave length, in figure 3 and the equation $\Gamma\left(\alpha, s, c^{\prime}\right)$ in figure 4 . 


\section{$\Gamma\left(\alpha, \mathbf{s}, \mathbf{c}^{\prime}\right)$}

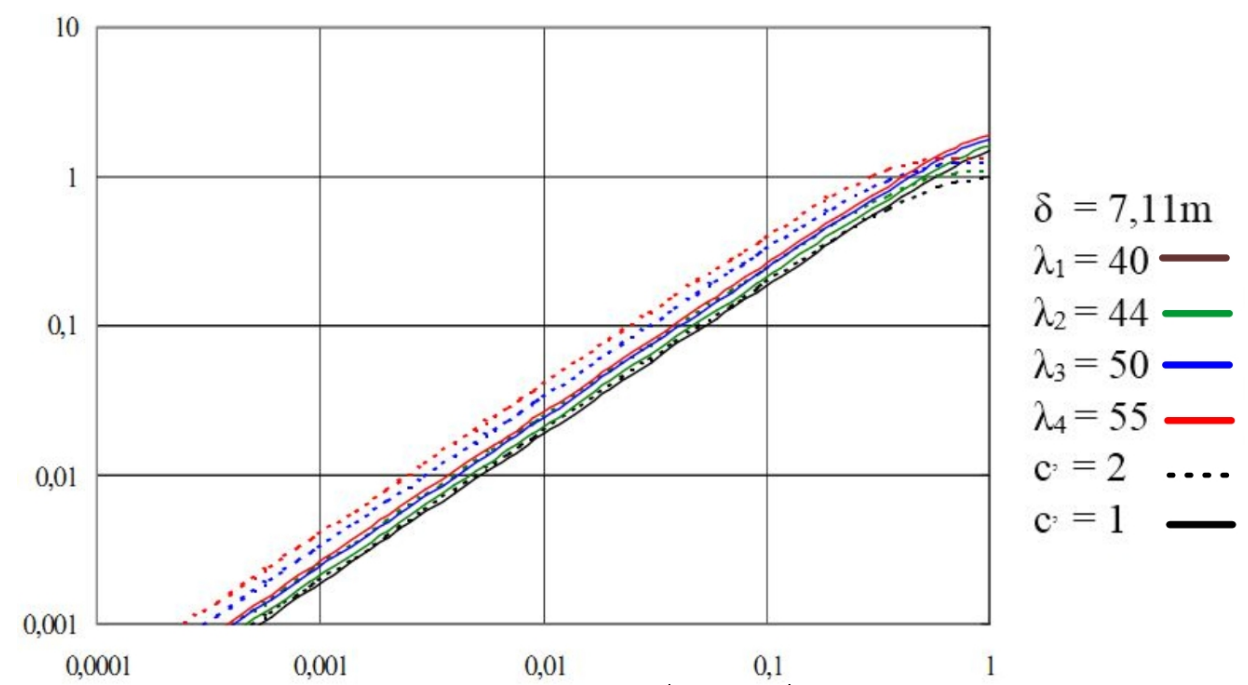

Figure 4 The variation's function $\Gamma\left(\alpha, s, c^{\prime}\right)$ for 4 wave length to progressive field

\section{Conclusions}

Taking into account the studied theoretical model, we searched the possibility for obtaining the electromagnetic pump effect (hydroelectromagnetic thruster), for seawater as electrolyte, having $\sigma=5 \mathrm{~S} / \mathrm{m}$ in a parallelepiped channel with the rectangular section, having three-phasic wrapping type "A".

Analyzing the expression of the traction force obtained by using the hydroelectromagnetic propeller with seawater the following conclusions imposed on:

- The value of the electromagnetic traction power is proportional with the effective square value of $\mathrm{J}_{\mathrm{So}}{ }^{2}$;
- The traction electromagnetic power with important values can be obtained only in parallelepiped channel with the width much more bigger than the inductor height;

- If the dimensions of the linear inductor increase $\lambda=2 \tau \times \mathrm{p}$ then more increases for the traction power $F_{x}$.

\section{Acknowledgements}

This work was a continuation of studies within the project "Research on the potential development of naval magnetohydrodynamic applications in seawater", Romanian Academy Grant no. $34 / 2006$.

\section{References}

[1] Dobref V.; Cantemir L.s.a.;. Possibilites de mise en mouvement nonconventionnelles des equipements et de la propultion navale. The $8^{\text {th }}$ World Conference Transport Research, Anvers, 1998.

[2] Dobref V.; Grozeanu S. Cercetari privind dezvoltarea fortelor propulsive in fluidele electroconductoare. A XXVII-a Sesiune de Comunicari Stiintifice cu participare Internationala, Bucureşti: Academia Tehnica Militara, 1997.

[3] Timotin A.; Panaitescu C. Asupra campului electromagnetic progresiv si fortelor din fluide conductoare. Studii si cercetari in energetica si electrotehnica, Tomul 23, nr.2, Bucureşti: Editura Academiei R.S.R, Bucuresti,1973, p. 289-292.

[4] Voldek A. Electromagnitnai transport jidko metala, iz metalurghia. Moscova, 1975. 